\title{
Editorial zum Themenheft
}

\section{„Auswirkungen des Bilanzrechtsmodernisierungsgesetzes (BilMoG) auf die Unternehmenssteuerung“6}

\author{
Carl-Christian Freidank
}

Online publiziert: 2. Juni 2010

(C) Springer-Verlag 2010

Das BilMoG vom 25. Mai 2009 hat insbesondere die Rechnungslegung und die Unternehmensüberwachung in der Bundesrepublik Deutschland grundlegend novelliert. Diese Gesetzesreform ist in Umfang und Reichweite mit den tiefgreifenden Änderungen durch das Bilanzrichtlinien-Gesetz im Jahre $1985 \mathrm{zu}$ vergleichen und führt zu elementaren Modifikationen der deutschen Rechnungslegung, die vor allem eine Neufassung zentraler Ansatz-, Bewertungs- und Konsolidierungsvorschriften bewirkt haben. Darüber hinaus sind von den Regelungen durch das BilMoG auch die Bereiche der Corporate Governance und der Abschlussprüfung betroffen, die von der Umsetzung der novellierten Achten EG-Richtlinie und der sog. EU-Änderungs-Richtlinie ausgelöst wurden.

Vor diesem Hintergrund beabsichtigt das vorliegende Themenheft mit seinen sechs Einzelbeiträgen, aus Sicht der Rechnungslegung, der Corporate Governance und des Controlling wichtige Auswirkungen des BilMoG auf die Unternehmenssteuerung zu untersuchen. Hierdurch sollen zum einen Gestaltungsstrategien, -potenziale und -instrumente aufgezeigt und kritisch analysiert werden, die von den Entscheidungsträgern künftig im Rahmen der Unternehmenspolitik genutzt werden können. Zum anderen zielen die Darlegungen darauf ab, Vorschläge zur Weiterentwicklung der durch das BilMoG ausgelösten Gesetzesänderungen zu erarbeiten.

Die ersten drei Beiträge des Themenheftes beschäftigen sich mit ausgewählten Novellierungen der Rechnungslegungsvorschriften durch das BilMoG, die von hoher Relevanz sowohl für die Rechnungslegungspolitik als auch für die Rechnungslegungsanalyse sind. Im Zentrum des Aufsatzes von Stefan Müller und Markus Kreipl steht zunächst eine Untersuchung des neuen abschlusspolitischen Potenzials, wobei

C.-C. Freidank $(\bowtie)$

Institut für Wirtschaftsprüfung und Steuerwesen, Lehrstuhl für Revisions- und Treuhandwesen, Universität Hamburg, Max-Brauer-Allee 60, 22765 Hamburg, Deutschland

e-mail: freidank@mba.uni-hamburg.de 
die Autoren als Beurteilungskriterium auf das internationale Postulat der Entscheidungsnützlichkeit von Informationen der Rechnungslegung zurückgreifen. Inge Wulf diskutiert anschließend die Streichung des pauschalen Aktivierungsverbotes für selbst geschaffene immaterielle Vermögensgegenstände des Anlagevermögens aus dem Blickwinkel der externen Rechnungslegungsanalyse. Zudem werden unter Rückgriff auf die Bilanztheorie Lösungsmöglichkeiten im Hinblick auf eine Modifikation der Unternehmensanalyse entwickelt. Der Beitrag von Marco Meyer fokussiert sodann die Neuregelung der Rechnungslegung latenter Steuern nach dem internationalen Temporary-Konzept. Im Einzelnen wird sowohl für den handelsrechtlichen Einzel- als auch den Konzernabschluss aufgezeigt, welche Auswirkungen der bilanzrechtliche Methodenwechsel im Rahmen der Ertragsteuerabgrenzung auf die Qualität eines Tax Reporting mit sich gebracht hat und künftig informationspolitisch genutzt werden kann.

Die beiden anschließenden Abhandlungen setzen sich mit Novellierungen des BilMoG auseinander, die die Corporate Governance betreffen. Zunächst gehen Reinhard Heyd und Michael Beyer der Frage nach, wie der neue Bericht zur Unternehmensführung als Publizitätsinstrument im Rahmen eines umfassenden Corporate Governance-Reporting aus Sicht der Principal-Agent-Theorie zu beurteilen ist. Patrick Velte und Stefan C. Weber beschäftigen sich im Folgenden mit Maßnahmen des BilMoG, die die Regulierung des Verhältnisses zwischen Vorstand, Aufsichtsrat und Abschlussprüfer betreffen. Auf der Grundlage theoretischer Erklärungsansätze für Koalitionsbildungen im Kontext der Corporate Governance wird verdeutlicht, dass es zusätzlicher normativer Schritte des Gesetzgebers bedarf, um mögliche Verbindungen zwischen Vorstand und Abschlussprüfer zulasten der Gehilfen- und Gatekeeper-Funktion des Letzteren zu verhindern.

Der abschließende Beitrag von Karsten Paetzmann und Thomas Kaspereit zeigt aus Sicht des wertorientierten Controlling auf, inwieweit die durch das BilMoG geänderten Rechnungslegungsvorschriften zu einer Attraktivitätssteigerung des Economic Value Added-Konzepts insbesondere für deutsche kapitalmarktferne Unternehmen geführt haben.

Hamburg, im April 2010

Carl-Christian Freidank 